\title{
How Long Hospitals Should Continue Contact Precautions for Multidrug Resistant Infections?
}

\author{
Masoud Mardani ${ }^{1},{ }^{*}$ \\ ${ }^{1}$ Infectious Diseases and Tropical Medicine Research Center, Shahid Beheshti University of Medical Sciences, Tehran, IR Iran \\ "Corresponding author: Masoud Mardani, Infectious Diseases and Tropical Medicine Research Center, Shahid Beheshti University of Medical Sciences, Tehran, IR Iran. E-mail: \\ drmasoudmardani@yahoo.com \\ Received 2018 April 01; Accepted 2018 April 20.
}

Keywords: Contact Precautions, Clostridium Difficile, Recommendations

Nowadays it has been proven that multidrug resistant and C. difficile infections have enjoyed virulent nature (1), thus, establishing policies on the duration of contact precaution to safety care for patients and prevent spread of these bacteria should be considered (2).

Recently, the society for health care epidemiology of America (SHEA) has published a guidelines, which precisely included all dimension and time of contact precaution (3).

These recommendations were published online on January 11, 2018 in the Infection Control and Hospital Epidemiology for one or more of the following organism, Methicillin-resistant Staphylococcus aureus (MRSA), vancomycin-resistant enterococci (VRE), clostridium difficile, and multi drug resistance entrobactrium.

There is a need for clinicians to examine the amount of time since the last positive sample, therefore, the guidance covers methicillin-resistant Staphylococcus aureus, vancomycin-resistant enterococci, and carbapenemresistant enterobacteriaceae. Specific recommendations are as follow:

For patients not accepting anti-microbials with movement against methicillin-safe $S$. aureus, the council prescribes utilizing negative screening societies to choose when to stop contact conventions. The ideal number of negative societies is indistinct, yet 1 to 3 are frequently utilized. Clinics might need to broaden contact precautionary measures for high-chance patients with ceaseless injuries and those from long haul mind offices. The perfect length of expansion is obscure, however, half a year is normal (3).

For exceptionally safe enterobacteriaceae, for example, carbapenemase-creating carbapenem-safe enterobacteriaceae, or enterobacteriaceae with few treatment alter- natives, healing centers need to keep contact precautionary measures uncertainly (3).

For C difficile contaminations, contact safety measures need to be proceeded for no less than 48 hours after the determination of looseness of the bowels. In addition, clinicians need to consider broadening insurances if $\mathrm{C}$ difficile disease rates stay high in spite of proper aversion and control measures (3).

With case of vancomycin-safe enterococci contamination, negative stool or rectal swab societies must be utilized to decide when to stop insurances. One to three negative cultures, no less than multi week separated, are regularly utilized (3).

There was inadequate proof to formally prescribe utilization of molecular testing to help direct choices on length of contact precautionary measures. It seems that polymerase chain reaction tests have better sensitivity compared with culture (3).

Hospitals need to carefully gauge their personal dangers, priorities, and sources while adopting coverage on duration of precautions, as costs and practicality of implementation fluctuate. Further more, the period of contact precautions can have a good-sized impact on the fitness of the patient, the hospital, and the network (3).

In Iran, due to lack of information about surveillance of hospital infections in the ICU and clinical wards preparing guideline in this regard has not been made yet. However, it seems that, this issue is one of the country research priorities in the field of infection control and prevention of antimicrobial resistance. Therefore, it is important that by preparing national guidelines, it could have important role in reducing the cost of hospital infections. 


\section{References}

1. Tokimatsu I, Shigemura K, Osawa K, Kinugawa S, Kitagawa K, Nakanishi N, et al. Molecular epidemiologic study of Clostridium difficile infections in university hospitals: Results of a nationwide study in Japan.J Infect Chemother. 2018. doi: 10.1016/j.jiac.2018.03.015. [PubMed: 29685855].

2. Le P, Nghiem VT, Mullen PD, Deshpande A. Cost-Effectiveness of Competing Treatment Strategies for Clostridium difficile Infection:
A Systematic Review. Infect Control Hosp Epidemiol. 2018;39(4):41224. doi: 10.1017/ice.2017.303. [PubMed: 29463339]. [PubMed Central: PMC5869164].

3. Banach DB, Bearman G, Barnden M, Hanrahan JA, Leekha S, Morgan DJ, et al. Duration of Contact Precautions for Acute-Care Settings. Infect Control Hosp Epidemiol. 2018;39(2):127-44. doi:10.1017/ice.2017.245. [PubMed: 29321078]. 